ISSN 0103-5150

Fisioter. Mov., Curitiba, v. 24, n. 2, p. 315-325, abr./jun. 2011

Licenciado sob uma Licença Creative Commons

\title{
Influência dos sistemas sensoriais na manutenção do equilíbrio em gestantes
}

\author{
Sensorial systems influence on the maintenance of the \\ balance in pregnant
}

\author{
Luana Mann $^{[\mathrm{a}]}$, Julio Francisco Kleinpaul ${ }^{[\mathrm{b}]}$, Clarissa Stefani Teixeira ${ }^{[\mathrm{cc}}$, Carlos Bolli Mota ${ }^{[\mathrm{d}]}$ \\ [a] Graduada em Educação Física e Mestranda em Educação Física, Universidade Federal de Santa Catarina (UFSC), \\ pesquisadora do Laboratório de Biomecânica (CDS), Florianópolis, SC - Brasil, e-mail: luanamann@gmail.com \\ [b] Mestre em Educação Física pela Universidade Federal de Santa Catarina (UFSC), professor de Educação Física do Centro \\ Universitário do Norte (UNINORTE), Manaus, AM - Brasil, e-mail: juliofk@gmail.com \\ [c] Graduada em Educação Física e Doutoranda em Engenharia da Produção, Universidade Federal de Santa Catarina (UFSC), \\ Florianópolis, SC - Brasil, e-mail: clastefani@gmail.com \\ [d] Doutor em Ciência do Movimento Humano, professor do curso de Educação Física da Universidade Federal de Santa \\ Maria (UFSM), Santa Maria, RS - Brasil, e-mail: bolli@cefd.ufsm.br
}

\section{Resumo}

Introdução: Existe um grande número de fatores interferindo na atuação do equilíbrio, porém a importância dada a cada um dos sistemas sensoriais durante a gestação ainda é desconhecida. 0 objetivo deste estudo foi investigar a influência dos sistemas visual, somatossensorial e vestibular no equilíbrio em diferentes fases da gestação. Método: Para a realização deste estudo foram avaliados dois grupos: grupo de gestantes (GG), e grupo de mulheres não gestantes (GC). 0 equilíbrio corporal foi mensurado por meio das seis condições do Teste de Organização Sensorial (TOS), utilizando-se uma plataforma de força AMTI ${ }^{\circledR}$. As variáveis relacionadas com a manutenção da estabilidade corporal foram a amplitude do deslocamento do centro de força nas direções ântero-posterior (COPap) e médio-lateral (COPml). Para comparação entre os grupos e entre os testes sensoriais, utilizou-se Anova One-Way, e para a identificação das diferenças utilizou-se o teste post hoc HSD de Tukey. O nível de significância adotado para todos os testes foi de 5\%. Resultados: As diferenças nas oscilações corporais são mais evidentes a partir do segundo trimestre de gestação, e a manipulação sensorial se mostra como fator agravante sobre essas oscilações. Conclusão: 0 processo de 
gestação influencia o equilíbrio em ambas as direções avaliadas (COPap e COPml) e as oscilações corporais aumentam com a dificuldade da tarefa (manipulação sensorial) e com o avançar da gestação.

Palavras-chave: Gestação. Equilíbrio. Sistemas sensoriais.

\section{Abstract}

Objective: There are a number of factors interfering with the performance of balance, however the importance given to each of the sensory systems during pregnancy is still unknown. The aim of this study was to investigate the influence of the visual, somatosensorial and vestibular system on the balance in the different stages of pregnancy. Methods: For the accomplishment of this study they were appraised two groups, being: group of pregnant women (GG), and non pregnant women's group (GC). The corporal balance was measured by the six conditions of the sensorial organization test (TOS), using a force platform AMTI ${ }^{\circledR}$. The variables related with the maintenance of the corporal stability were the displacement of the center of force in the anterior-posterior (COPap) and medium-lateral directions (COPml). For comparison between groups and between the sensory tests a One-Way Anova was used, and to identify the differences the post hoc HSD Tukey test was used. The significance level adopted for all tests was 5\%. Results: Differences in body movements are more obvious from the second trimester of pregnancy, and the sensory manipulation is shown as an aggravating factor on them. Conclusion: The process of pregnancy influences the balance in both directions evaluated (COPap and COPml) and body oscillations increase with the difficulty of the task (sensory manipulation) and the progress of pregnancy.

Keywords: Pregnancy. Balance. Sensory systems.

\section{Introdução}

A gravidez é um processo fisiológico compreendido pela sequência de adaptações ocorridas no corpo da mulher a partir da fertilização. A preparação do corpo para a gestação envolve ajustes dos mais variados sistemas (1) e pode ser considerada um estado de saúde que envolve mudanças fisiológicas iguais ou maiores do que as que acompanham muitos estados patológicos (2).

As alterações são advindas de mudanças hormonais que aumentam a frouxidão ligamentar (3) e mudanças biomecânicas (4) que provocam modificações estruturais na estática e dinâmica do esqueleto da gestante. Como exemplo destas considerações podem ser citadas as mudanças músculo-esqueléticas no corpo da gestante, como o constante crescimento do útero, o aumento no peso corporal e o tamanho das mamas, os quais contribuem para o deslocamento do centro de gravidade para cima e para frente, podendo acentuar e promover uma anteversão pélvica e um consequente desequilíbrio. Além disso, há uma compensação da hiperlordose lombar causada, de forma a utilizar base de suporte maior (5) e modificações nos padrões considerados normais para a marcha e para o equilíbrio $(4,6)$.

Desordens de equilíbrio representam uma preocupação de saúde pública crescente por causa da associação com quedas e danos a elas relacionados. Os estudos internacionais demonstram que, durante a gravidez, uma em cada quatro mulheres relata quedas, taxa esta comparável a pessoas idosas com mais de 65 anos $(7,8)$. As quedas podem resultar em sérios problemas de saúde e representam 17$39 \%$ dos traumas maternos (7) e respondem por $3-7 \%$ das mortes fetais $(9,10)$. Porém, estudos nacionais ainda se encontram abrandados no que tange a essas estatísticas.

A manutenção do equilíbrio requer uma complexa integração das informações sensoriais advindas dos sistemas visual, somatossensorial e vestibular relativas à posição do corpo e a habilidade para gerar respostas motoras apropriadas para controlar o movimento corporal $(11,12)$. Ao sistema vestibular cabe a sensibilidade de detectar as acelerações lineares e angulares, enquanto o sistema somatossensorial é composto por vários receptores que percebem a posição e a velocidade de todos os segmentos corporais, seu contato com objetos externos, 
inclusive o chão, e a orientação da gravidade (13). Já a informação visual é baseada nas características externas do ambiente. Com a perfeita integração desses sistemas, no cérebro, mais especificamente com tronco encefálico e cerebelo, juntamente com memórias de experiências prévias, a correta postura do indivíduo é determinada e, portanto, qualquer disfunção nestes sistemas pode desencadear sintomas de falta de equilíbrio $(14,15)$.

O Teste de Organização Sensorial é uma importante ferramenta para análise do controle postural, uma vez que analisa a contribuição relativa dos receptores somatossensoriais, visuais e vestibulares na estabilidade global do indivíduo, indicando qual dos sistemas utilizados para a manutenção do equilíbrio é responsável pela instabilidade (padrões de distribuição sensorial), assim como sua capacidade de manter o equilíbrio com informações sensoriais erradas (visão preferencial) (16).

Mesmo que existam muitos estudos realizados com o equilíbrio (17-19), não foram encontrados estudos que vislumbrem a relação entre a gestação e os sistemas sensoriais responsáveis pela estabilidade. Além disso, a atuação e a importância dada a cada um dos sistemas sensoriais durante a gestação ainda são desconhecidos, tanto pela literatura nacional quanto pela internacional. A obtenção de conhecimentos como esses é importante para a formulação da prescrição de tratamentos de reabilitação mais adequados para compensar o sistema que se encontra debilitado, permitindo a valorização objetiva deste. Dessa forma, o objetivo do presente estudo foi investigar a influência dos sistemas visual, somatossensorial e vestibular no equilíbrio em diferentes fases da gestação.

\section{Materiais e métodos}

Este estudo foi aprovado pelo comitê de ética da instituição na qual foi desenvolvido, sob número de processo 23081.003779/2008-23, no qual foram cumpridos os princípios éticos contidos na declaração de Helsinque, além do atendimento à legislação vigente.

Para a realização do estudo foram avaliados dois grupos selecionados de forma não probabilística intencional, sendo: grupo de gestantes (GG), composto por 15 gestantes, e grupo de mulheres não gestantes (GC), composto por 10 mulheres sedentárias.
Para a composição dos grupos, foram convidadas mulheres gestantes e não gestantes da comunidade santamariense. Após os primeiros contatos, elas, antes de qualquer coleta, assinaram o Termo de Consentimento Livre e Esclarecido concordando em participar do estudo. Por meio da ficha de anamnese foram realizados os procedimentos para inclusão e exclusão e para coleta de dados prévios como idade, peso, estatura corporal, prática de exercícios físicos, ocorrência de quedas e problemas de saúde.

Os critérios de inclusão do estudo foram: ter idade entre 18 e 42 anos, não ter problemas músculoesqueléticos e/ou vestibulares, não possuir patologias como diabetes, hipertensão arterial, obesidade ou doenças infecciosas, ou queixas de tontura. Estes critérios foram verificados por meio do questionário Dizziness Handicap Inventory (DHI), conhecido como DHI Brasileiro, validado para o português brasileiro por Castro (20) e publicado por Castro et al. (21). Para o GG, as mulheres deveriam estar entre a $12^{\text {a }}$ e a $32^{\text {a }}$ semana de gestação, e para o GC, as mulheres não poderiam estar grávidas, nem relatar a ocorrência de dor lombar, já que esta síndrome afeta o equilíbrio corporal $(18,22)$. Em função de indicações na literatura sobre diferenças no controle postural em diferentes fases da gestação $(5,23)$, o grupo de gestantes foi subdividido em três subgrupos: G1, de 13 a 16 semanas de gestação; G2, de 22 a 25 semanas de gestação (ambos grupos formados por gestantes do segundo trimestre, estando respectivamente no período inicial e final deste); e G3, de 28 a 29 semanas de gestação, período medial do terceiro trimestre. Essa divisão foi definida com intervalo máximo intragrupo de três semanas de gestação, buscando aproximar as características das gestantes e tornar os dados mais confiáveis para comparações.

O número de indivíduos, a média e o desvio padrão da idade, do peso e da estatura corporal dos diferentes grupos do estudo estão dispostos na Tabela 1.

A avaliação do equilíbrio corporal e suas relações com o sistema visual, somatossensorial e vestibular seguiu o teste de organização sensorial (TOS) proposto por Nashner (24). As seis condições do teste são:

- TOS 1: visão normal e plataforma de apoio fixa sob os pés. Neste teste avaliam-se os sistemas visual, somatossensorial (proprioceptivo) e vestibular; 
Tabela 1 - Média (X) e o desvio-padrão (S) da idade, do peso e da estatura corporal dos diferentes grupos do estudo e número de indivíduos em cada grupo

\begin{tabular}{|c|c|c|c|c|c|c|c|}
\hline \multirow[b]{2}{*}{ Grupo } & \multirow{2}{*}{$\begin{array}{l}\text { Número de } \\
\text { indivíduos }\end{array}$} & \multicolumn{2}{|c|}{ Idade (anos) } & \multicolumn{2}{|c|}{ Peso corporal (N) } & \multicolumn{2}{|c|}{ Estatura $(\mathrm{m})$} \\
\hline & & $x$ & $S$ & $x$ & S & $x$ & $S$ \\
\hline $\mathrm{GC}$ & 10 & 19,70 & 5,43 & 588,22 & 202,93 & 1,66 & 0,07 \\
\hline G1 & 5 & 20,20 & 1,13 & 633,18 & 174,17 & 1,58 & 0,05 \\
\hline G2 & 5 & 32,04 & 11,10 & 624,12 & 112,30 & 1,60 & 0,10 \\
\hline G3 & 5 & 29,76 & 3,54 & 769,03 & 173,08 & 1,74 & 0,05 \\
\hline
\end{tabular}

Fonte: Dados da pesquisa.

- TOS 2: visão ausente e plataforma de apoio fixa sob os pés. Este teste avalia os sistemas proprioceptivo e vestibular;

- TOS 3: visão normal, plataforma de apoio fixa sob os pés e referência (cabine visual) oscilante. Este teste avalia o sistema proprioceptivo, vestibular e, sobretudo, o visual;

- TOS 4: visão normal e plataforma de apoio oscilante sob os pés. Este teste avalia principalmente o sistema proprioceptivo;

- TOS 5: visão ausente e plataforma de apoio oscilante sob os pés. Este teste avalia os sistemas proprioceptivo e vestibular, em condições de sobrecarga, graças à eliminação da aferência visual e à movimentação da plataforma;

- TOS 6: visão normal com referência (cabine visual) e suporte de apoio oscilante. A avaliação é referente aos sistemas proprioceptivo, visual e vestibular.

A mensuração das variáveis referentes ao equilíbrio corporal foi realizada utilizando-se uma plataforma de força 0R6-5 AMTI $^{\circledR}$ (Advanced Mechanical Technologies, Inc.). Para mensurar o conflito nos sistemas relacionados com o equilíbrio corporal foram utilizadas uma cabine e uma superfície de espuma, validadas por Castagno (25). Para a avaliação, o indivíduo é posicionado sobre a plataforma de força dentro de uma cabine de $1 \mathrm{~m}^{2}$, com altura de $2 \mathrm{~m}$, confeccionada com suporte de ferro desmontável, envolta por um tecido de algodão com listras horizontais, claras e escuras de $10 \mathrm{~cm}$ cada uma. A cabine é um sistema mecânico simples e move-se $20^{\circ}$ manualmente para frente e para trás durante a realização dos
TOS 3 e 6. Para realizar a manipulação do sistema somatossensorial, nos TOS 4, 5 e 6, foi utilizada uma almofada de $10 \mathrm{~cm}$ de espessura, de $50 \mathrm{~cm}$ x $50 \mathrm{~cm}$, entre os pés do indivíduo e a plataforma de força. Todas as seis condições testadas foram realizadas em posição ortostática, com os pés descalços e os olhos fixos na horizontal. 0 tempo de aquisição dos dados para cada tentativa foi de 20 segundos, após a estabilização visual do centro de força (COP), a uma frequência de aquisição de $100 \mathrm{~Hz}$. Os testes foram selecionados de forma randomizada, por meio de sorteio, para cada indivíduo.

As variáveis relacionadas com a manutenção da estabilidade corporal foram a amplitude do deslocamento do centro de força nas direções ântero-posterior (COPap) e médio-lateral (COPml). Estas variáveis foram mensuradas pela plataforma de força e dadas pelas seguintes equações:

$$
\begin{aligned}
& \mathrm{COP}_{a p}=[(M y-h \cdot F x) / F z]_{\max }-[(M y-h \cdot F x) / F z]_{\min } \\
& \mathrm{COP}_{m l}=[(M x-h \cdot F y) / F z]_{\max }-[(M x-h \cdot F y) / F z]_{\text {min }}
\end{aligned}
$$

Em que:

$C O P_{a p}=$ coordenada do centro de força na direção ântero-posterior;

$C O P_{m l}=$ coordenada do centro de força na direção médio-lateral;

$M x=$ momento em torno do eixo ântero-posterior;

$M y=$ momento em torno do eixo médio-lateral;

$F X$ = componente ântero-posterior da força de reação do solo;

Fy = componente médio-lateral da força de reação do solo; 
$F z=$ componente vertical da força de reação do solo; $h=$ distância da superfície até o centro geométrico da plataforma de força.

0 peso corporal foi mensurado pela própria plataforma de força e a estatura por um estadiômetro da marca Welmy ${ }^{\circledR}$ com resolução de 0,5 cm.

As variáveis (COPap e COPml) foram comparadas considerando os diferentes grupos (GC x G1 x G2 x G3) em cada TOS (TOS 1 a 6) com o intuito de verificar a influência do período gestacional sobre a estabilidade. Além disso, as variáveis COPap e COPml foram comparadas considerando os seis TOS (TOS 1 x TOS 2 x TOS $3 \times$ TOS 4 x TOS 5 x TOS 6) nos diferentes grupos, com o objetivo de verificar a influência dos sistemas sensoriais (visual, somatossensorial e vestibular) nos diferentes grupos (GC, G1, G2 e G3).

Os dados foram submetidos à estatística descritiva. A normalidade dos dados foi verificada através do teste de Shapiro-Wilk, que mostrou que os dados podem ser considerados como tendo distribuição normal. Para comparação entre os grupos e entre os TOS utilizou-se Anova One-Way, e para a identificação das diferenças utilizou-se o teste post hoc HSD de Tukey. 0 nível de significância adotado para todos os testes foi de $5 \%$. Para estas análises foi utilizado o pacote estatístico SPSS ${ }^{\circledR}$ for Windows ${ }^{\circledR}$ versão 11.5.

\section{Resultados}

Os resultados mostram que com o avançar da gestação ocorrem maiores instabilidades corporais em ambas as variáveis (COPap e COPml), em comparação ao grupo de não gestantes, conforme indicam as Figuras 1 e 2. As oscilações apresentaram maiores diferenças considerando o COPap nos TOS 2,5 e 6, visto que todos os grupos mostraram-se estatisticamente diferentes entre si (Figura 1). Já no sentido médio-lateral ( $\mathrm{COPml}$ ) as diferenças ocorreram principalmente entre G1 (13 a 16 semanas de gestação) e G2 (22 a 25 semanas de gestação) nos TOS 1, 2 e 4 (Figura 2).

Com relação à influência dos sistemas sensoriais, observou-se que o maior número de diferenças se iniciou no G2. No G3 (28 a 29 semanas de gestação) todos os testes (de 1 a 6 ) mostraram diferenças estatisticamente entre si na direção ântero-posterior (COPap) (Figura 3). Já na direção médio-lateral as diferenças mostraram-se significativas quando os TOS 5 e 6 foram analisados em função dos demais TOS (Figura 4).

Em relação à comparação entre os TOS em cada grupo, o G1 apresentou os maiores valores de oscilação para o COPap no TOS 6, sendo que este diferiu de todos os demais. Este comportamento se repetiu para o COPml no TOS 5. No G2, as maiores oscilações do COPap ocorreram no TOS 6, sendo que todos os valores de TOS diferiram entre si, com exceção dos TOS 2 e 4, enquanto que as maiores oscilações do COPml ocorreram no TOS 2 , sendo que este não diferiu estatisticamente dos TOS 4, 5 e 6. No G3, as maiores oscilações também ocorreram no TOS 6, sendo que este diferiu de todos os demais, tanto no COPap quanto no COPml, conforme indicam as Figuras 3 e 4.

De forma geral, pode-se dizer que não foram encontrados padrões que permitam determinar a importância, bem como a preferência entre os sistemas sensoriais para cada teste, de acordo com o avançar do período gestacional. Os valores apresentados na Figura 1 são referentes às comparações dos grupos GC, G1, G2 e G3 em cada um dos seis TOS, considerando o sentido ântero-posterior (COPap) e na Figura 2 considerando o sentido médio-lateral (COPml).

Os resultados referentes às comparações dos seis TOS (TOS 1, TOS 2, TOS 3, TOS 4, TOS 5 e TOS 6) em cada grupo, considerando o sentido ânteroposterior (COPap), estão ilustrados na Figura 3, e o sentido médio-lateral (COPml), na Figura 4.

\section{Discussão}

O objetivo do presente estudo foi investigar a influência dos sistemas visual, somatossensorial e vestibular no equilíbrio em diferentes fases da gestação. De forma geral, os resultados encontrados indicam que quanto maior o número de sistemas sensoriais manipulados, maiores são as oscilações corporais das gestantes, uma vez que o TOS 6 apresentou os maiores valores de oscilação corporal. Assim, pode-se dizer que os resultados do presente estudo corroboram com a literatura consultada $(17,19,26)$ que aponta aumento significativo nos valores das variáveis de equilíbrio com aumento da dificuldade da tarefa, que no caso do TOS 6 permite a manipulação do sistema vestibular, por meio da oscilação da cabine visual, e do sistema 


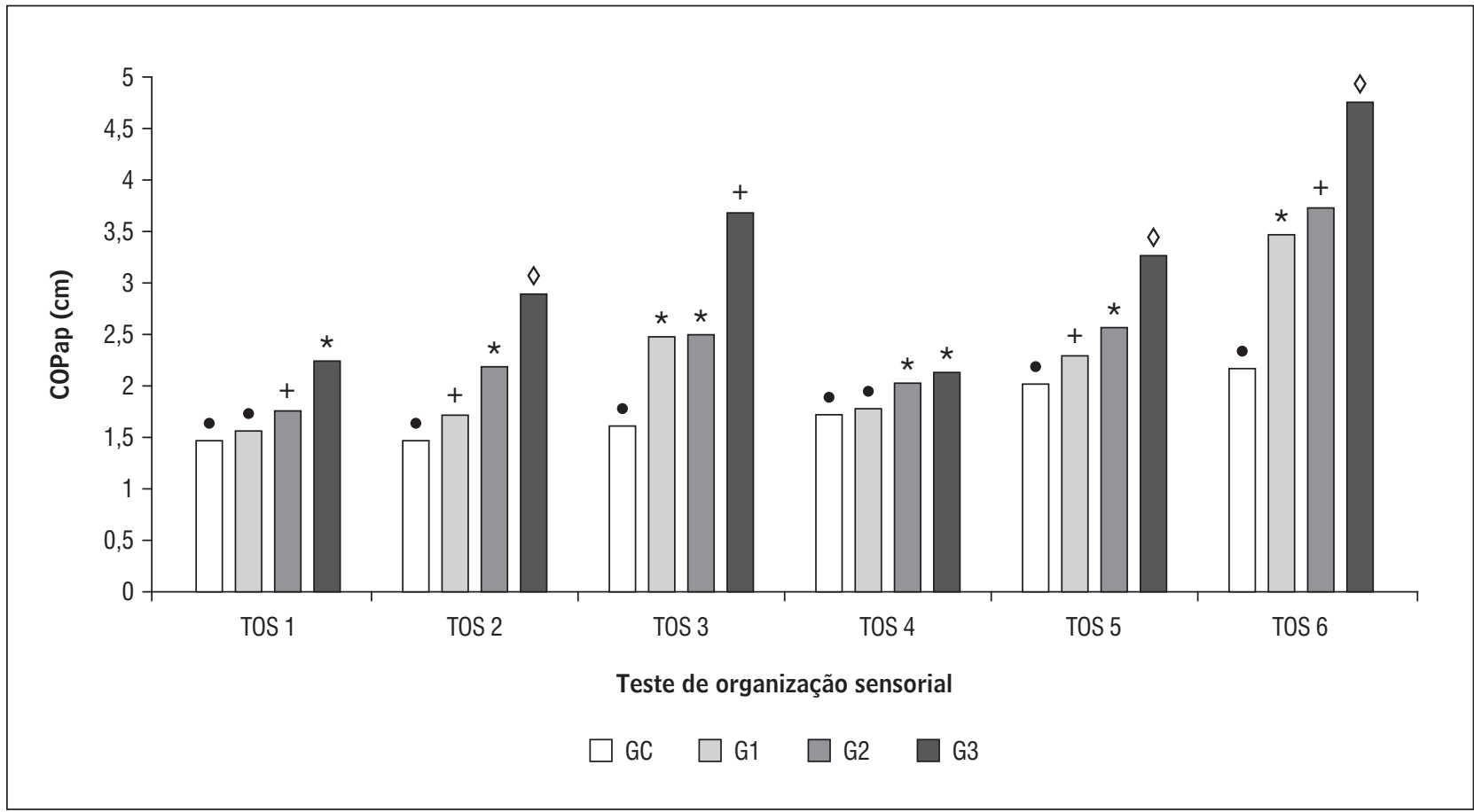

Figura 1 - Deslocamento do Centro de Força na direção ântero-posterior (COPap, em cm) para os seis Testes de Organização Sensorial (TOS) nos quatro grupos (GC, G1, G2 e G3) avaliados. • + * Símbolos diferentes indicam diferenças estatisticamente significativas entre os grupos $(p<0,05)$ (Tukey)

Fonte: Dados da pesquisa.

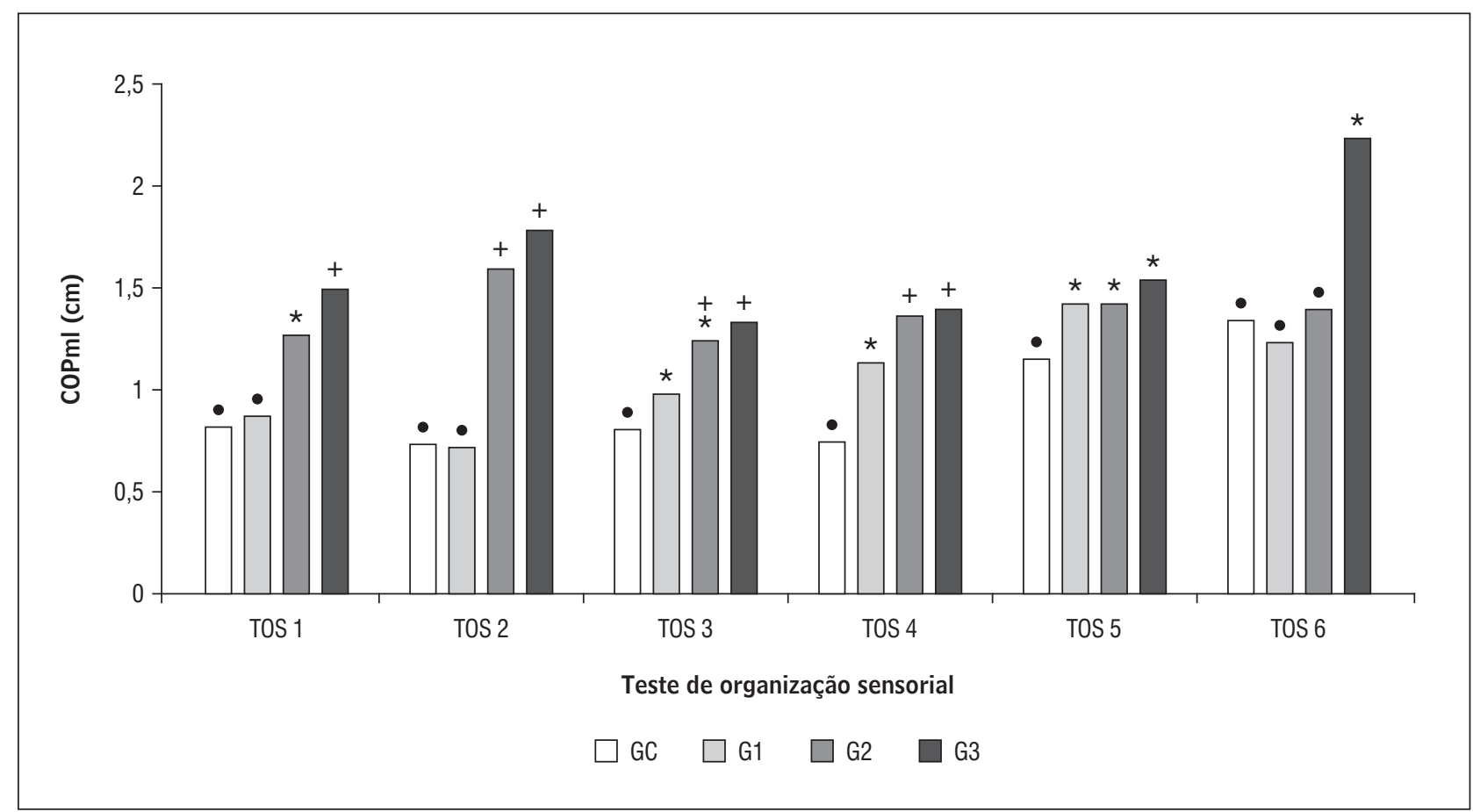

Figura 2 - Deslocamento do Centro de Força na direção médio-lateral (COPIm, em cm) para os seis Testes de Organização Sensorial (TOS) nos quatro grupos (GC, Gl, G2 e G3) avaliados. •+* Símbolos diferentes indicam diferenças estatisticamente significativas entre os grupos $(p<0,05)$ (Tukey)

Fonte: Dados da pesquisa. 


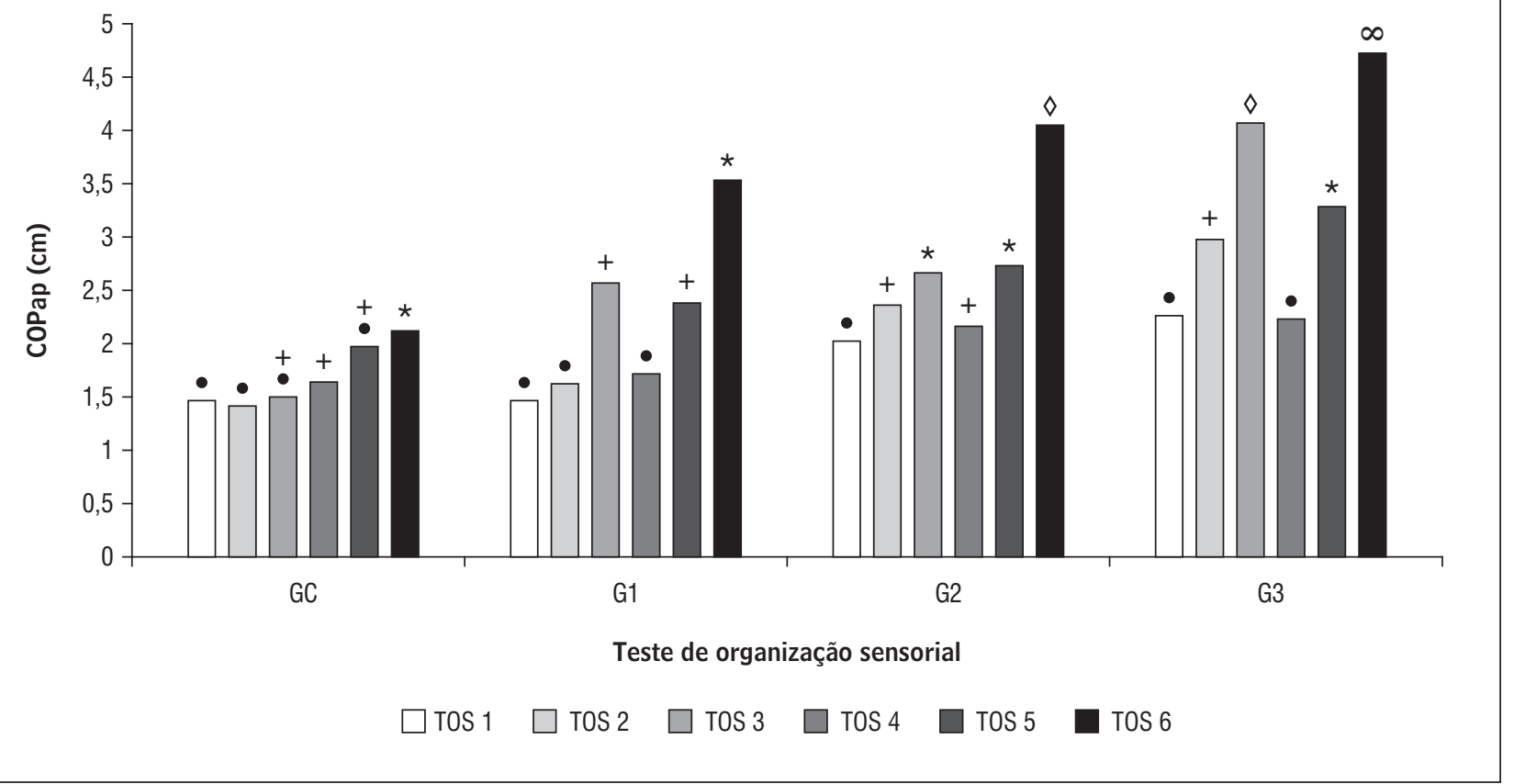

Figura 3 - Deslocamento do Centro de Força na direção ântero-posterior (COPap, em cm) para os quatro grupos (GC, Gl, G2 e G3) nos seis Testes de Organização Sensorial (TOS) avaliados. • +* Símbolos diferentes indicam diferenças estatisticamente significativas entre os TOS ( $p<0,05)$ (Tukey)

Fonte: Dados da pesquisa.

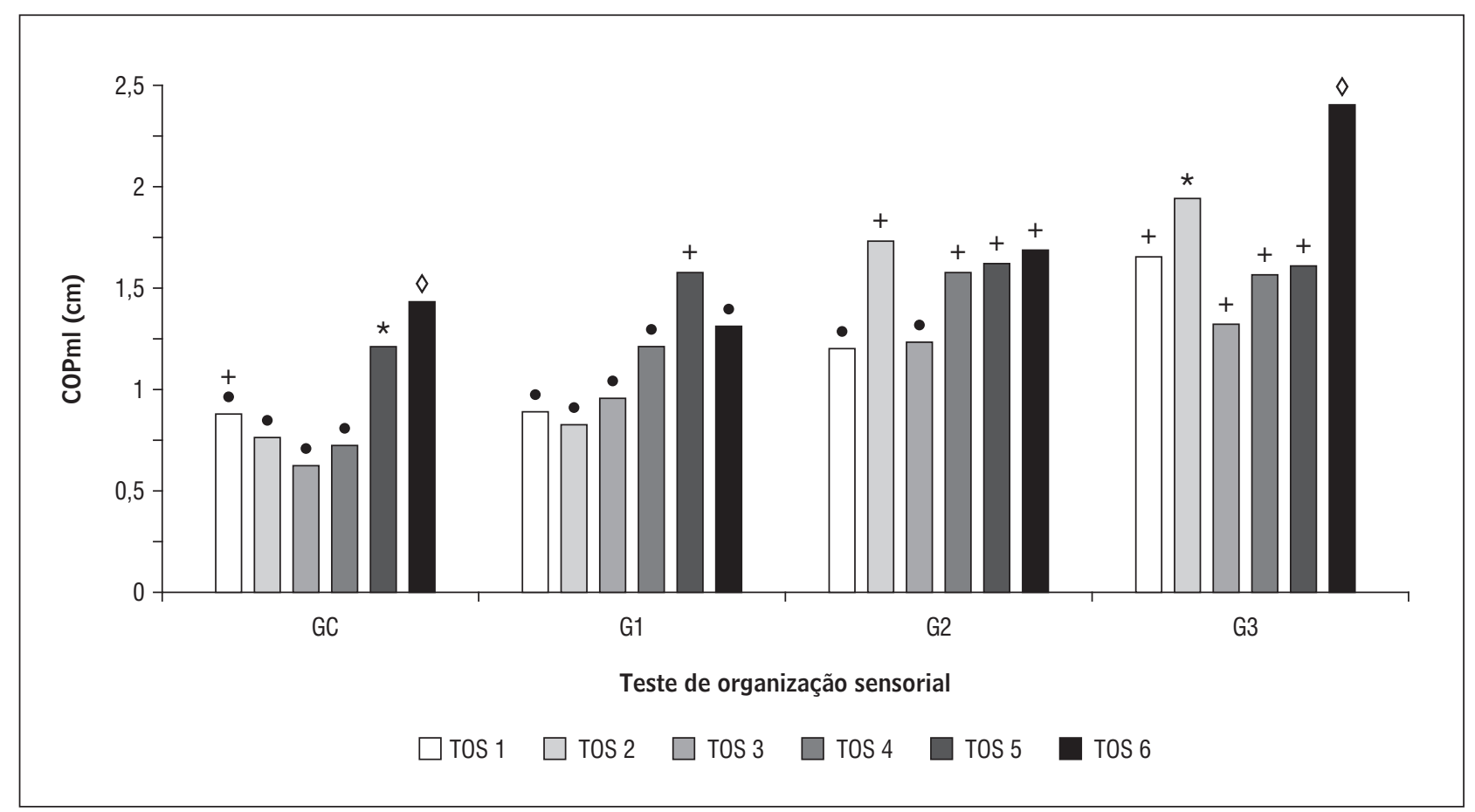

Figura 4 - Deslocamento do Centro de Força na direção médio-lateral (COPml, em cm) para os quatro grupos (GC, G1, G2 e G3) nos seis Testes de Organização Sensorial (TOS) avaliados. $\bullet+*$ Símbolos diferentes indicam diferenças estatisticamente significativas entre os TOS $(p<0,05)$ (Tukey)

Fonte: Dados da pesquisa. 
somatossensorial, por meio da almofada entre os pés e o solo.

Freitas Júnior e Barela (27) afirmam que quando o cenário visual é movimentado, como no TOS 3 e no TOS 6, ocasionando um conflito vestibular, os indivíduos produzem oscilações corporais correspondentes ao movimento do cenário visual para manter o quadro de referência estabelecido, o qual implica a manutenção da estabilidade do cenário visual projetado na retina. Neste sentido, os resultados do presente estudo (Figura 3) confirmam estas considerações, visto que quando os TOS de 1 a 3 foram comparados, o TOS 3 tendeu a ter maiores oscilações no sentido ântero-posterior, indicando a importância do sistema vestibular no equilíbrio corporal de gestantes. Quando houve manipulação do sistema somatossensorial, como no caso dos TOS 4 a 6, a mesma tendência ocorreu, ou seja, maiores oscilações foram encontradas quando o sistema vestibular foi acometido (TOS 6) e quando houve ausência da visão (TOS 5) para o sentido ânteroposterior. Já em relação ao $\mathrm{COPml}$ não houve uma tendência entre os grupos, apenas no G3 ocorreu um aumento de oscilação quando o cenário visual foi movimentado (TOS 6) indicando importância do sistema vestibular, enquanto que com a manipulação dos sistema somatossensorial o TOS 4 foi significativo para o G2 e o TOS 5 para o G1, conforme indica Figura 4.

Especificamente em relação à manipulação proprioceptiva, Baloh et al. (28) indicam que diminuindo a informação dos pés e dos tornozelos, com utilização da almofada, o indivíduo é forçado a confiar mais nos sinais vestibulares para o controle do equilíbrio, principalmente com os olhos fechados. Esta consideração vai ao encontro dos resultados do presente estudo, uma vez que, no geral, para todos os grupos de gestantes, o TOS 5 se diferiu dos demais TOS no sentido ântero-posterior e no sentido médio-lateral, apenas no G1 ocorreu esta diferença. Segundo Black e Paloski (29), neste teste específico a informação sensorial avaliada é referente ao sistema vestibular, pois o visual não está em ação e o proprioceptivo está alterado.

Segundo Paloski et al. (30), com a ausência da visão e manipulação proprioceptiva, observa-se a diminuição da habilidade do sistema vestibular para discernir a orientação da gravidade e manter a postura. Desta forma, as diferenças encontradas no sentido ântero-posterior são melhores entendidas, visto que no TOS 5 todos os grupos diferiram entre si (Figura 1), estando o GC com melhor estabilidade e o G3 com a pior, ou seja, com maiores desequilíbrios.

Os resultados do presente estudo vão ao encontro do de Butler et al. (31), uma vez que as diferenças entre os TOS 1 e 2 ocorreram a partir do G2, denotando que com o avançar da gestação ocorre aumento da dependência visual. Segundo Patel et al. (17), o efeito da visão é mais importante na direção ântero-posterior, com aumento da complexidade da tarefa. Em suma, os autores concluem que durante a postura estática, sobre superfícies estáveis, ocorrem dois diferentes efeitos: a diminuição da habilidade de distribuir as forças com precisão para manter a devida orientação corporal e a dificuldade de responder corretivamente com a natureza da tarefa submetida.

No caso do TOS 2, que também apresenta supressão da visão, as diferenças ocorreram em todos os grupos, considerando a direção ântero-posterior. Com relação ao sentido médio-lateral, as diferenças ocorreram a partir do G3, ou seja, 28 a 29 semanas de gestação. Assim, notou-se que a visão é importante, mesmo estando os sistemas proprioceptivo e vestibular presentes para o desenvolvimento da tarefa. Os resultados do presente estudo demonstraram que o sistema visual é importante para manutenção da estabilidade corporal, já que a ausência da visão mostrou aumento das oscilações posturais em praticamente todos os grupos avaliados (Figuras 3 e 4), concordando com Latash (32), que afirma que a visão é o sistema sensorial que o corpo mais confia nas tarefas de manutenção da postura e de movimento. Possivelmente, em virtude desses fatos há grande influência da visão em tarefas de manutenção do equilíbrio e controle postural.

Para Sanz et al. (11), a informação visual suprimida ou manipulada não afeta o controle postural em indivíduos "sadios" desde que os demais sistemas recebam uma informação adequada para fazer a compensação. Assim, entende-se o fato de o GC apresentar menores valores de oscilações do COP e, consequentemente, melhores valores de equilíbrio corporal.

De maneira geral, o aumento da dificuldade da tarefa com a manipulação de mais sistemas sensoriais já foi indicado por outros estudos. Ruwer, Rossi e Simon (33), por exemplo, indicaram que quando dois sistemas estiverem ausentes e/ou manipulados, 
a dificuldade na manutenção do controle postural é aumentada. Já Wade et al. (34) explicam que as oscilações posturais aumentam na medida em que a distância entre o observador e a cena visual aumenta. No presente estudo essas afirmações ficaram evidentes à medida que o TOS 6 se diferiu dos demais em todos os grupos de gestantes, apresentando os maiores valores de desequilíbrios na direção ântero-posterior (Figura 3). Da mesma forma, pode-se citar o TOS 3, que se mostrou com diferenças significativas comparando-se o GC com os demais grupos, tanto na direção ântero-posterior quanto na direção médio-lateral (Figuras 1 e 2). Nos outros testes as diferenças em relação ao GC ocorreram principalmente a partir das semanas finais do segundo trimestre (G2 - 22 a 25 semanas de gestação) (Figuras 1 e 2).

No estudo de Jang, Hsiao e Hsiao-Wecksler (5) as oscilações no sentido médio-lateral permaneceram estáveis durante a gravidez, mas aumentadas após o parto, o que, segundo os autores, ocorre em função do aumento da base de apoio durante a gestação para manutenção da estabilidade. Estas indicações se refletem nos resultados encontrados pelo presente estudo, que mostrou que no sentido médio-lateral o aumento da dificuldade da tarefa associou-se principalmente às fases finais da gestação (Figura 2).

Porém, não foram encontrados comportamentos padrões de oscilações tanto considerando os grupos quanto considerando os TOS. Estes resultados podem se dever ao número reduzido de indivíduos avaliados. Mesmo assim, a realização do presente estudo é importante, visto que estudos como este não foram encontrados na literatura e dados brasileiros ainda mostram-se abrandados.

Além dessas considerações, as explicações para a falta de padrão dos dados podem estar voltadas para a habilidade do sistema nervoso mudar discretamente a fonte principal de informação sensorial para controlar a postura e, quando faz a transição de uma fonte de percepção para outra, o faz de forma abrupta e usa uma informação sensorial de cada vez (35). Além disso, em situações em que alguma informação não está disponível, seja em função de déficits em um dos sistemas sensoriais ou em função do contexto da tarefa, o sistema de controle postural ainda é capaz de detectar o posicionamento corporal com base nas informações disponíveis, selecionando as informações sensoriais mais relevantes dentro do contexto para manter a postura ereta (35-37).
Os resultados do presente estudo permitem identificar que o grupo que se apresentou mais acometido, visto o número de diferenças encontradas, foi o G3, que se mostrou com as maiores oscilações, principalmente considerando o sentido ântero-posterior (Figura 3). Além disso, o mesmo grupo mostrou-se diferente dos demais considerando o COPap nos TOS 1, 2, 3, 5 e 6 (Figura 1). No sentido médio-lateral, as diferenças ocorreram principalmente comparando os indivíduos do GC com os demais, sendo que este apresentou os melhores valores de equilíbrio corporal nos TOS 3, 4 e 5 (Figura 2). Da mesma forma, o GC mostrou-se com maior estabilidade para o COPap nos TOS 2, 3, 5 e 6.

0 fato de que mulheres grávidas apresentem diminuída sua habilidade de manter o equilíbrio na posição em pé já vem sendo indicado pela literatura (38). Os prejuízos, quando acometidos os sistemas vestibular e somatossensorial, como no caso do TOS 6, foram causados principalmente para as gestantes em período gestacional mais avançado, ou seja, no G3 (28 a 29 semanas de gestação). Além disso, quando considerado o sentido destas oscilações, a direção ântero-posterior foi a que apresentou maiores oscilações. Da mesma forma, quando considerada a direção médio-lateral, o G3 também se mostrou com os maiores valores de oscilação, o que indica maiores desequilíbrios. Estes achados corroboram com os estudos de Jang, Hsiao e HsiaoWecksler (5), Butler et al. (8), Mann et al. (23) e Butler at al. (24), que indicam aumento das instabilidades corporais (maior oscilação do COP) com o avançar da gestação.

De forma geral, o presente estudo indicou que as diferenças em relação a não gestantes ocorreram a partir do início do segundo trimestre (G1) para o sentido ântero-posterior (Figura 1), corroborando com Jang, Hsiao e Hsiao-Wecksler (5), que reportaram aumento da oscilação corporal na direção ântero-posterior durante a gestação e diminuição dos valores de oscilação após o parto, e ao estudo de Butler et al. (31). Butler et al. (31) explicam que o ganho de peso mais gradual característico do primeiro trimestre da gestação explica as não diferenças no equilíbrio corporal no primeiro trimestre gestacional, em comparação a não gestantes. A partir do segundo e terceiro trimestres ocorre um ganho de peso maior, o que pode explicar a diminuição da estabilidade postural durante essas fases da gestação. 


\section{Conclusões}

Ao se investigar a influência dos sistemas visual, somatossensorial e vestibular no equilíbrio em diferentes fases da gestação, verificaram-se maiores oscilações corporais com a dificuldade da tarefa. Na ausência da visão ou com manipulação vestibular, somadas ao acometimento do sistema somatossensorial, a manutenção da estabilidade corporal foi dificultada e os maiores valores de desequilíbrio foram encontrados, como no caso dos TOS 5 e 6. Além disso, houve influência do período gestacional considerando os diferentes testes, e o grupo com maiores instabilidades foi o G3 (28 a 29 semanas de gestação).

As oscilações foram diferentes considerando o COPap nos TOS 2, 5 e 6, visto que todos os grupos mostraram-se estatisticamente diferentes entre si. Já no sentido médio-lateral (COPml) as diferenças ocorreram principalmente entre G1 (13 a 16 semanas de gestação) e G2 (22 a 25 semanas de gestação) nos TOS 1, 2 e 4.

Com relação à influência dos sistemas sensoriais, observou-se que o maior número de diferenças se iniciou no G2, sendo que no G3 todos os testes (de 1 a 6) mostraram diferenças estatísticas entre si na direção ântero-posterior (COPap). Já na direção médio-lateral, as diferenças mostraram-se significativas quando os TOS 5 e 6 foram analisados em função dos demais TOS.

\section{Referências}

1. Mantle J, Polden M. Fisioterapia em ginecologia e obstetrícia. São Paulo: Santos; 2005.

2. Santos GM. Avaliação biomecânica do andar durante a gestação [dissertação]. Santa Maria: Universidade Federal de Santa Maria; 1998.

3. Marnach ML, Ramin KD, Ramsey PS, Song SW, Stensland JJ, An KN. Characterization of the relationship between joint laxity and maternal hormones in pregnancy. Obstet Gynecol. 2003;101(2):331-5.

4. Rodacki CL, Fowler NE, Rodacki AL, Birch K. Stature loss and recovery in pregnant women with and without low back pain. Arch Phys Med Rehabil. 2003; 84(4):507-12.
5. Jang J, Hsiao KT, Hsiao-Wecksler ET. Balance (perceived and actual) and preferred stance width during pregnancy. Clin Biomech. 2008;23(4):468-76.

6. Carpes FP, Kleinpaul JF, Mann L, Griebeler D, Mota CB. Women able-bodied gait kinematics during and post pregnancy period. Rev Bras Biomec. 2008; 9(16):33-40.

7. Dunning K, LeMasters G, Levin L, Bhattacharya A, Alterman T, Lordo K. Falls in workers during pregnancy: risk factors, job hazards, and high risk occupations. Am J Ind Med. 2003;44(6):664-72.

8. Butler EE, Colón I, Druzin M, Rose J. An investigation of gait and postural balance during pregnancy. Gait Post. 2006a;24(Suppl. 2):128-9.

9. Connolly AM, Katz VL, Bash KL, McMahon MJ, Hansen WF. Trauma and pregnancy. Am J Perinatol. 1997; 14(6):331-6.

10. Weiss HB, Songer TJ, Fabio A. Fetal deaths related to maternal injury. JAMA. 2001;286(15):1863-8.

11. Sanz EM, Guzman B, De Cerverón CC, Baydal JM. Análisis de la interacción visuo-vestibular y la influencia visual en el control postural. Acta Otorinolaringol Esp. 2004;55:9-16.

12. Sturnieks DL, George R, Lord SR. Balance disorders in the elderly. Clin Neurophysiol. 2008;38(6):467-78.

13. Winter D. ABC of balance during standing and walking. Waterloo, Ontario: Waterloo biomechanics; 1995.

14. Shumway-Cook A, Woollacott MH. Motor control: theory and practical applications. Maryland: Williams \& Wilkins; 1995.

15. Spirduso WW, Francis KL, Macrae PG. Motor control, coordinations and skill. In: Spirduso WW, Francis KL, Macrae PG, editors. Physical dimensions of aging. Champaign, IL: Human Kinectics; 1995. p. 152-183.

16. Ronda JM, Galvañ B, Monerris E, Ballester F. Asociación entre sintomas clínicos y resultados de la posturografía computadorizada dinámica. Acta Otorinolaringol Esp. 2002;53(4):252-5.

17. Patel M, Fransson PA, Lush D, Gomez S. The effect of foam surface properties on postural stability assessment while standing. Gait Post. 2008;28(4):649-56. 
18. Carpes FP, Reinehr FB, Mota CB. Effects of a program for trunk strength and stability on pain, low back and pelvis kinematics, and body balance: a pilot study. J Bodyw Mov Ther. 2008;12(1):22-30.

19. Harringe ML, Halvorsen K, Renström P, Werner S. Postural control measured as the center of pressure excursion in young female gymnasts with low back pain or lower extremity injury. Gait Post. 2008; 28(1):38-45.

20. Castro ASO. Dizziness Handicap inventory: adaptação cultural para o português brasileiro, reprodutibilidade e comparação com os resultados à vestibulometria [dissertação]. São Paulo: Universidade Bandeirante de São Paulo; 2003.

21. Castro ASO, Gazzola JM, Natour J, Ganança FF. Brazilian version of the Dizziness Handicap Inventory. Pró-Fono. 2007;19(1):97-104.

22. Mann L, Kleinpaul JF, Weber P, Mota CB, Carpes FP. Efeito do treinamento de Isostretching sobre a dor lombar crônica - um estudo de casos. Motriz. 2009; 15(1):50-60.

23. Mann L, Kleinpaul JF, Teixeira CS, Lopes LFD, Konopka CK, Mota CB. Gestação: equilíbrio corporal, dor lombar e quedas. Rev Bras Biomec. 2009;9(18):14-21.

24. Nashner LM. Computerized dynamic posturography. In: Jacobson GP, Newman CW, Kantush JM, editors. Handbook of balance testing. St. Louis, Missouri: Mosby-Year Book; 1993. p. 280-307.

25. Castagno LA. Anew method for sensory organization tests: the foam-laser dynamic posturography. Rev Bras Otorrinolaringol. 1994;60(4):287-96.

26. Baydal-Bertomeu JM, Guillem RB, Soler-Gracia C, Moya MFP, Prat JM, Guzmán RB. Determinación de los patrones de comportamiento postural en población sana española. Acta Otorinolaringol Esp. 2004;55:260-9.

27. Freitas Júnior PB, Barela JA. Alterações no funcionamento do sistema de controle postural de idosos: uso da informação visual. Rev Port Cien Desp. 2006; 6(1):94-105.

28. Baloh RW, Jacobson KM, Enrietto JA, Corona S, Honrubia V. Balance disorders in older persons: quantification with posturography. Otolaryngol Head Neck Surg. 1998;119:89-92).
29. Black FO, Paloski WH. Computerized dynamic posturography: what have we learned from space? Otolaryngol Head Neck Surg. 1998;118(3 Pt 2):S45-51.

30. Paloski WH, Wood SJ, Feiveson AH, Black FO, Hwang EY, Reschke MF. Destabilization of human balance control by static and dynamic head tilts. Gait Post. 2006;23(3):315-23.

31. Butler EE, Colón I, Druzin ML, Rose J. Postural equilibrium during pregnancy: decreased stability with an increased reliance on visual cues. Am J Obstet Gynecol. 2006;195(4):1104-8.

32. Latash ML. Neurophysiological basis of human movement. Champaign: Human Kinetics; 1997.

33. Ruwer SL, Rossi AG, Simon LF. Equilíbrio no idoso. Rev Bras Otorrinolaringol. 2005;71(3):298-303.

34. Wade MG, Lindquist R, Taylor JR, Treat-Jacobson D. Optical flow, spatial orientation, and the control of posture in the elderly. J Gerontol B Psychol Sci Soc Sci. 1995;50(1):P51-P58.

35. Mccollum C, Shupert CL, Nashner LM. Organizing sensory information for postural control in altered sensory environments. J Biol. 1996;180(3):257-70.

36. Horak FB, Macpherson JM. Postural orientation and equilibrium: exercise: regulation and integration of systems multiple. In: Rowell LB, Shepard JT, editors. Handbook of physiology. New York: Oxford University Press; 1996. p. 255-92.

37. Oie KS, Kiemel T, Jeka JJ. Multisensory fusion: simultaneous reweighting of vision and touch for control of human posture. Brain Res Cogn Brain Res. 2002; 14(1):164-76.

38. Macevilly M, Buggy D. Back pain and pregnancy: a review. Pain. 1996;64(3):405-14.

Recebido: 16/08/2010

Received: 08/16/2010

Aprovado: 28/12/2010

Approved: 12/28/2010 